\title{
THERMODYNAMICS APPLIED. WHERE? WHY?
}

\author{
Gerard Hirs \\ University of Twente, Faculty of Mechanical Engineering \\ P.O. Box 217, 7500 AE Enschede \\ The Netherlands \\ Phone ++31-53-4892561, Fax ++31-53-48936663 \\ E mail g.g.hirs@wb.utwente.nl
}

\begin{abstract}
In recent years thermodynamics has been applied in a number of new fields leading to a greater societal impact. The paper gives a survey of these new fields and the reasons why these applications are important. In addition it is shown that the number of fields could be even greater in the future and could lead to savings in human effort, use of land and water and of energy and natural resources. Achieving these savings is a contribution to a sustainable society.
\end{abstract}

\section{INTRODUCTION}

Traditionally energy conversion and some industrial processes such as air separation have been subjected to thermodynamic analysis. Later other industrial processes such as steel making and bulk chemical production have been added. Thermodynamic analysis has been shown to be useful on different levels: systems, processes, process units and components. Technology development on all levels speeds up if the results of thermodynamic analysis prove to be favorable. Combined heat and power is a recent example. Other types of combined and integrated production are being considered. Two types, one on municipal waste processing and another on synthesis gas and electricity production will be dealt with here.

From the start of thermodynamics applied in engineering it became clear that analysis should encompass both the first and the second law of thermodynamics. This has led to the development of energy/exergy analysis. The development of the method still continues. An example is the design of heat exchangers where the traditional design method is based on the first law only and the newly developed method on both first and second law.

Largely outside the field of thermodynamics a new type of analysis and assessment has been developed: LCA or Life Cycle Analysis. The analysis is in most cases environment oriented and systematically produces an inventory of all input and output attributable to the object being analyzed. The object can be a service, a product, a plant, etc. It is analyzed during its entire life cycle from cradle to grave. The result of the analysis is a list of quantities or equivalent quantities that contribute to a number of environmental effects: resource depletion, greenhouse, ozone, smog, radiation, etc. Remarkable differences between products can be shown to occur. This will be demonstrated by giving results for a porcelain mug and a plastic cup. 
In the now usual LCA's energy is not well accounted for. For this reason Exergetic Life Cycle Analysis, ELCA, has been further developed. It will be shown to yield an exergy loss for the complete life cycle of mug and cup.

The consequences of recycling are implicitly dealt with in LCA and ELCA. Routes with and without recycling can be considered by comparing the lists of quantitative environmental effects and the exergy losses. An example will show the difference between combustion of waste wood and its use replacing gypsum as board material.

It is well known that results of thermodynamic analysis can be translated into monetary cost. In exergo-economics and thermo-economics the translation only takes place in the utilization phase of the life cycle and not in the phases before and after utilization. For those phases only monetary costs of equipment and services are taken into consideration. Such costs are estimated on the basis of information from industry or empirical formulae derived from this information. This approach will be shown to contradict life cycle thinking.

\section{THERMODYNAMICS IN ENERGY CONVERSION}

This is perhaps the oldest field where second law analysis was applied and exergy analysis developed. Exergy analysis is carried out in the development and the design phase. An interesting new development is monitoring the operation of a plant and determining exergy losses of plant components from moment to moment. The case to be presented here has been selected because it shows a thermodynamically favorable integration of the use of a low quality fuel, municipal waste, and a high quality fuel, natural gas. It also shows how the thermodynamic performance can be judged.

In Western Europe municipal solid waste (MSW) incineration is combined with steam generation and electricity production. The thermodynamic performance is poor because the peak steam temperature has to be kept below $400 \mathrm{C}$ in order to prevent damage to the boiler. Moreover the stack gas clean up takes much of the electricity that is produced. Design values for the thermodynamic performance are 20-25 percent, in operation it is more like 15-20 percent, based on lower heating value.

Improving the performance without extensive technological development proved possible by integrating the waste incineration with a combined cycle fired by natural gas.

Case 1 represents the combination where the combustion air of the combined cycle and the waste incineration are in parallel and where the steam produced by the waste incineration is heated from $400 \mathrm{C}$ to above $500 \mathrm{C}$ in the heat recovery steam generator of the combined cycle.

Case 2 is different by using the exhaust from the combined cycle as secondary air for the incineration.

Case 3 represents the combination where the combined cycle and the waste incineration are placed in series: the exhaust gas from the combined cycle is used as inlet air for the waste incineration. In this case the heat recovery steam generator is reduced to a super heater.

Case 4 is different from Case 3 by adding boiler surface.

The results of the analysis in Figure 1 show a significant increase in efficiency.

The drawn line represents separate production of electricity by combined cycle $(0$ percent MSW fraction of total fuel input) and waste incineration (100 percent MSW fraction). The dots above this line represent the four cases, all indicate a gain in efficiency. Case 1 and 4 are very close, Case 2 coincides with 1 . Case 3 appears to obtain the greatest gain with respect to separate production. It can be seen as an advantage that the MSW fraction is highest in that case. In Figure 2 the reference case is included. 
For all cases MSW fractions, efficiencies and heat exchange surface are shown. It appears that Case 3 with combined cycle and incineration in series has the smallest increase in heat surface area when compared with pure incineration.

Naturally there are other reasons that influence the selection between alternatives. In Holland the combined cycle had to be capable of operating independently of waste incineration. Then Case 1 is the obvious choice. The plant is now operating since three years without problems.

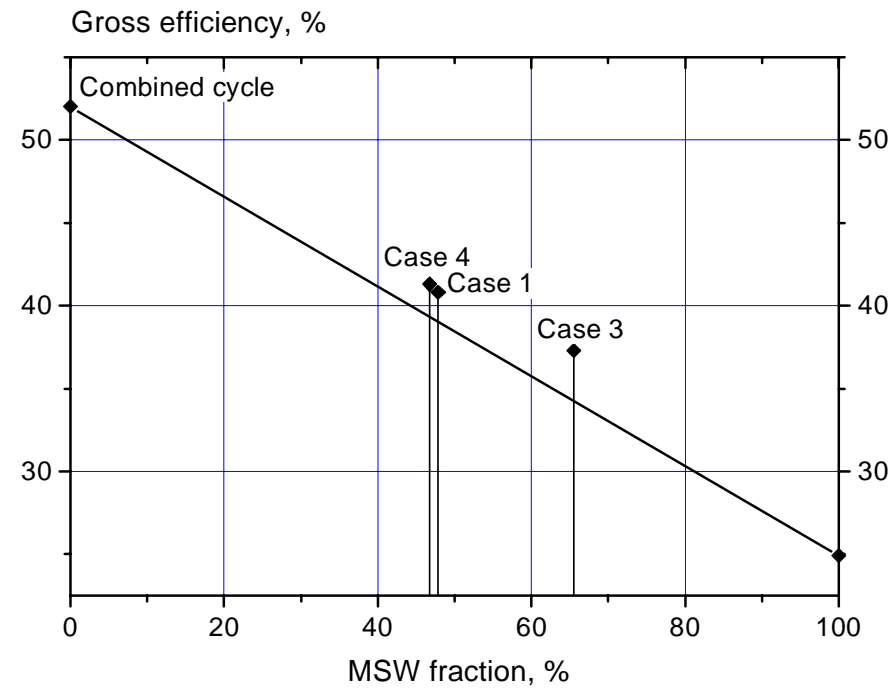

Figure 1 The MSW fraction in the total fuel input versus gross efficiency

Gross efficiency Surface area

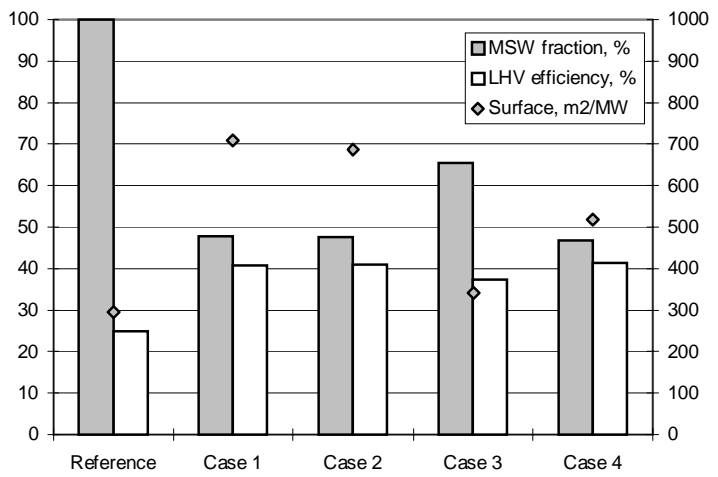

Figure 2 Comparison of the MSW fraction in the total fuel input, the gross plant Efficiency(LHV), and the specific boiler surface area

\section{INTEGRATED PRODUCTION OF BULK AND ELECTRICITY CHEMICALS}

Natural gas is one of the main sources for bulk chemicals. Its importance will grow if more natural gas becomes available. Synthesis gas, a mixture of carbon monoxide and hydrogen, is produced from natural gas and forms the basis for many bulk chemicals such as methanol, ammonia, diesel fuel, etc. In many processes more than half of the exergy 
content of the natural gas is destroyed for producing the bulk chemical. An example of such a process is the Shell Middle Distillate Synthesis (SMDS).

The three main causes of the loss are:

- production of synthesis gas by steam reforming, an endothermic reaction in a directly fired furnace

- production of synthesis gas by partial oxidation, an exothermal reaction at low temperature and pressure

- other directly fired furnaces in the production process where heat is needed

Development is switching from steam reforming to partial oxidation and increasing attention to heat integration. However integrated production with electricity is hardly explored. The reason probably is that it would require the development of specific gas turbine technology.

Synthesis gas production in a gas turbine system is illustrated in Figure 3.

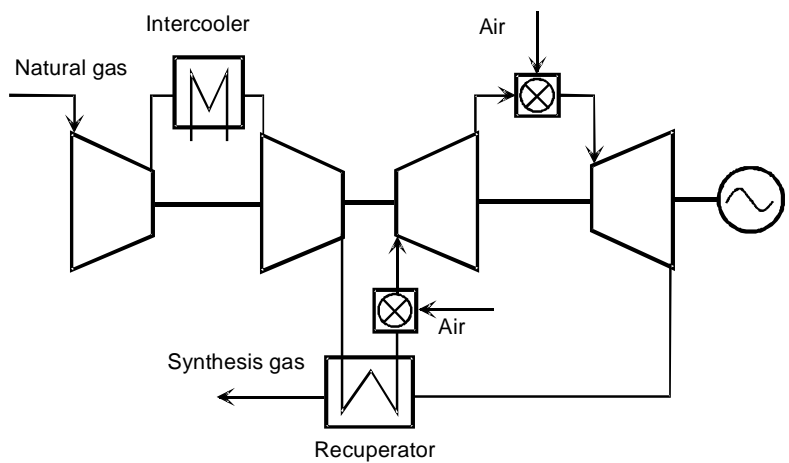

Figure 3 Synthesis gas production in gas turbine system

The natural gas is compressed in two steps. The incoming air, or oxygen with a carrier gas such as carbon dioxide, is also compressed in two steps (not shown in the figure). The natural gas is partially oxidized in two chambers in order to keep the peak temperature below values that are acceptable to gas turbine practice. Before entering the second turbine the natural gas is oxidized as follows:

$$
2 \mathrm{CH}_{4}+\mathrm{O}_{2} \rightarrow 2 \mathrm{CO}+4 \mathrm{H}_{2}
$$

The quantity of oxygen used in this partial oxidation process is $1 / 4$ of full oxidation. Contrary to normal gas turbine practice there is no excess of oxidant to control peak temperatures in the reaction chambers.

However in the case of partial oxidation an excess of natural gas can be used for this purpose. Alternatively a carrier gas can be used such as nitrogen, carbon dioxide and water. The choice will be influenced by the bulk chemical to be produced: e.g. fertilizer, carbon fibre or methanol. It can be concluded that the partial oxidation process goes hand in hand with a reduction of the exergy content. The difference in exergy between methane and synthesis gas is available for work or electricity. If gas turbine technology for full oxidation can be translated to partial oxidation, it is expected that a thermodynamic efficiency of 50-60 percent can be achieved.

Perhaps the most interesting aspect of this gas turbine system is that it does not produce any emission to the environment. The exhaust gas of the system is the product

\section{HEAT EXCHANGER DESIGN}


In heat exchanger design the first law of thermodynamics is still regarded as the ultimate physical insight. On the basis of the first law the effectiveness of a heat exchanger has been defined and has been adopted in many textbooks. It is defined by the following equation:

$$
\varepsilon=\frac{C_{h}\left(T_{h, \text { in }}-T_{h, o u t}\right)}{C_{\text {min }}\left(T_{h, \text { in }}-T_{c, \text { in }}\right)}=\frac{C_{c}\left(T_{c, o u t}-T_{c, \text { in }}\right)}{C_{\text {min }}\left(T_{h, \text { in }}-T_{c, \text { in }}\right)}
$$

where $\mathrm{C}_{\min }=\mathrm{C}_{\mathrm{c}}$, when $\mathrm{C}_{\mathrm{h}}>\mathrm{C}_{\mathrm{c}}$ and $\mathrm{C}_{\min }=\mathrm{C}_{\mathrm{h}}$, when $\mathrm{C}_{\mathrm{h}}<\mathrm{C}_{\mathrm{c}}$.

It is assumed that there are no heat losses to the environment. Any attempt to also take pressure loss into account in the effectiveness is fruitless.

It seems useful to base heat exchanger design on both the first and the second law of thermodynamics.

The thermodynamic performance is determined by the input exergy of the stream that is cooled and the output exergy of the stream that is heated. The difference between these two is the exergy loss due to loss in quality of the transferred heat. The exergy losses due to the flow resistance and due to the production of the heat exchanger hardware can be included. However, these are omitted here in order to make a direct comparison possible between the thermodynamic efficiency and the effectiveness. The thermodynamic efficiency is defined as the ratio between output and input exergy. In Figure 4 results are presented.

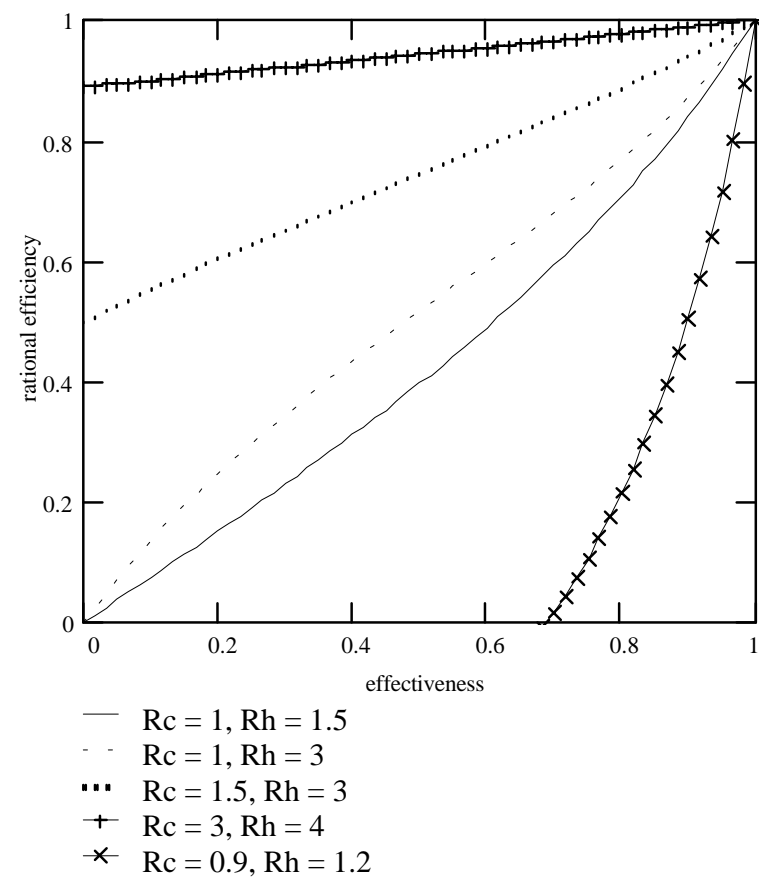

Figure 4: Thermodynamic efficiency versus effectiveness for various cold and hot temperature ratios for $\mathrm{C}_{\mathrm{h}}=\mathrm{C}_{\mathrm{c}}$

The thermodynamic efficiency is on the vertical axis and the effectiveness on the horizontal axis. For different values of $\mathrm{Rc}$ and $\mathrm{Rh}$ lines are shown. $\mathrm{Rc}$ is the ratio of temperatures at the inlet of the cold stream, Tc,in, and the environmental temperature, To. $\mathrm{Rh}$ is the ratio of temperatures at the inlet of the hot stream, Th,in and environmental 
temperature. The figure is representative for a countercurrent heat exchanger with equal thermal capacities of hot and cold fluid. For a temperature ratio for the cold flow of unity $(\mathrm{Rh}=1)$ and a temperature ratio of the hot stream $(\mathrm{Rh})$ of 1.5 and 3 the thermodynamic efficiency and the effectiveness are shown to be roughly the same. At higher values of the temperature ratio, Rc $>1$, the thermodynamic efficiency is clearly above the effectiveness. At lower values of the temperature ratio, $\mathrm{Rc}<1$, the thermodynamic efficiency is lower than the effectiveness which is due the fact that the exergy below To is completely lost. It clearly demonstrates that hot streams should in practice be cooled as much as possible by streams from the environment and not by means of a cooling machine.

The conclusion is that the effectiveness is no suitable yardstick for heat exchanger design and operation. Thermodynamic performance and thermodynamic efficiency quantify the loss in terms of exergy or in terms of a ratio between exergy output and input. For the uninitiated it should be remembered that an exergy loss can be translated into a quantity of gas, oil or an amount of money.

\section{LIFE CYCLE ANALYSIS}

The life cycle of a product, a plant or a service is characterized by three phases: production, operation and disposal. The life cycle can be refined and extended by adding: mining, transportation, assembly, maintenance, recycling, etc. The object of the life cycle analysis should be clearly defined and include the function of the object, its quantified constitutive parts, production routes, energy conversion routes during the different phases of the life cycle, etc. The most important aspect of an LCA is its objective. Its objective should be expressed in terms of environment, employment, money, exergy or any other objective that seems relevant. Until now most LCA's are environment oriented. In fact, the LCA-method has been developed by environment-conscious scientists. However, life cycle thinking has entered also other societal domains. An important example is that several larger firms are concentrating on their core business and are contracting out the complete life cycle of entire plants: design, erection, operation and disposal. Their ultimate goal is to sell the product and achieve minimum life cycle cost of the plant. Life cycling thinking has led to environmental benefits in industry even in those cases where the objective was not explicitly environment oriented. In the case of process plant, the focus of the attention of the firm has shifted away from minimum investment cost to minimum cost of investment plus operation plus disposal. This proved to be a benefit to the environment in many cases.

The overall objective of an LCA can focus on more than one single objective. Frequently, two objectives, e.g. exergy and cost, are counteracting. However, allowing for a small increase in the monetary life cycle cost can generate an enormous decrease in exergetic life cycle cost. Having two objectives leads to a weighing problem and requires that both an exergetic LCA and a monetary LCA are carried out.

When entrepreneurs take more risk, e.g. by installing a new higher efficiency gas turbine, lower monetary cost and lower exergetic cost go hand in hand. If everything goes well!

When entrepreneurs have to prevent specific emissions higher monetary and exergetic costs coincide.

The question to be discussed here is how an environment oriented LCA and an exergetic ELCA are related. As an example a disposable polystyrene cup and a procelain mug are compared, both being used 3000 times.

The basic difference is the fact that the cups are disposed of after once being used and that the mug is washed after use. The cup is not recycled. The mug is washed in a detergent containing 30 percent phosphates. The results are displayed in Figure 5. The display is in 
units per thousand PE, $1 \mathrm{PE}$ is the emission from all sources per inhabitant per year. The spokes of Figure 5 clearly show the relative magnitude of the emissions for every environmental impact. The magnitude of the longest spoke for depletion is 6 PE. It appears that some emissions are negligible, such as those due to pesticides, heavy metals and carcinogenic materials. It also appears that the emissions are a factor of 2 or more greater for the disposable cup than for the mug. It should be mentioned that the acidification and the smog are mainly due to refineries which were not assumed to have clean up systems. The depletion effect is measured in terms of exergy loss due to both fuels and feed stock for materials. The exergy balances are shown in Figures 6 and 7.

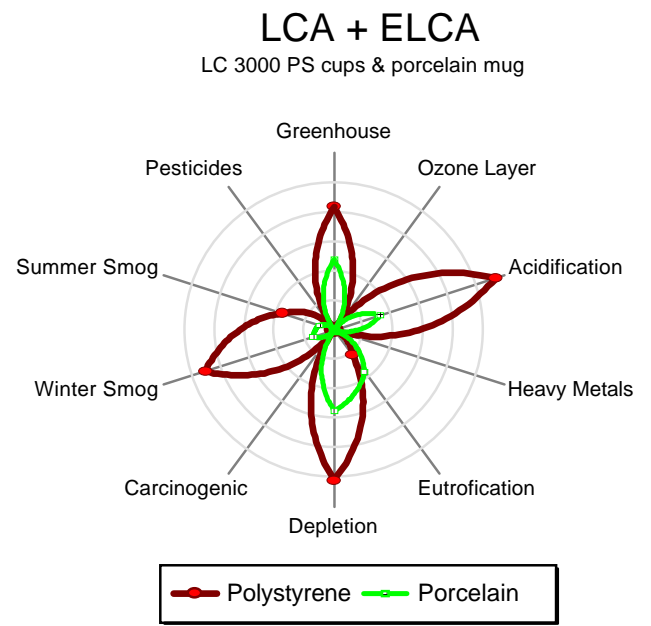

Figure 5 The normalised environmental effects, including depletion, each circle is, starting from the center, an increase of 0.001 PE.

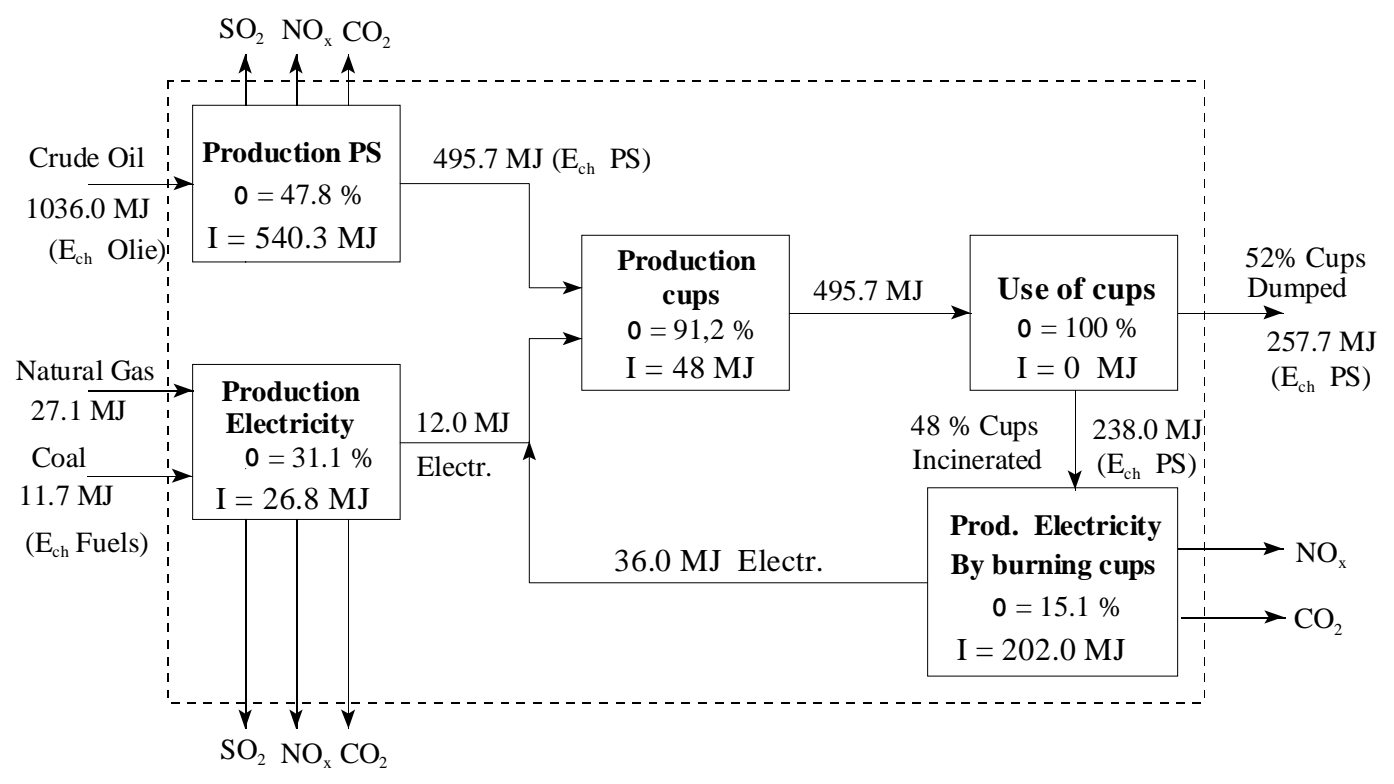

Figure 6 Exergy diagram of the life cycle of the 3000 disposable PS cups 
When studying environmental problems by way of LCA and ELCA it becomes evident that the emissions can be greatly reduced by accepting some additional exergy loss or by a further depletion of natural resources, see Figure 8.

It can be shown that acidification and smog do not require much additional exergy loss. The main part of the additional loss is taken by preventing the greenhouse effect, about 25 percent. This explains why $\mathrm{CO} 2$ removal is not economically justified at this moment in time.

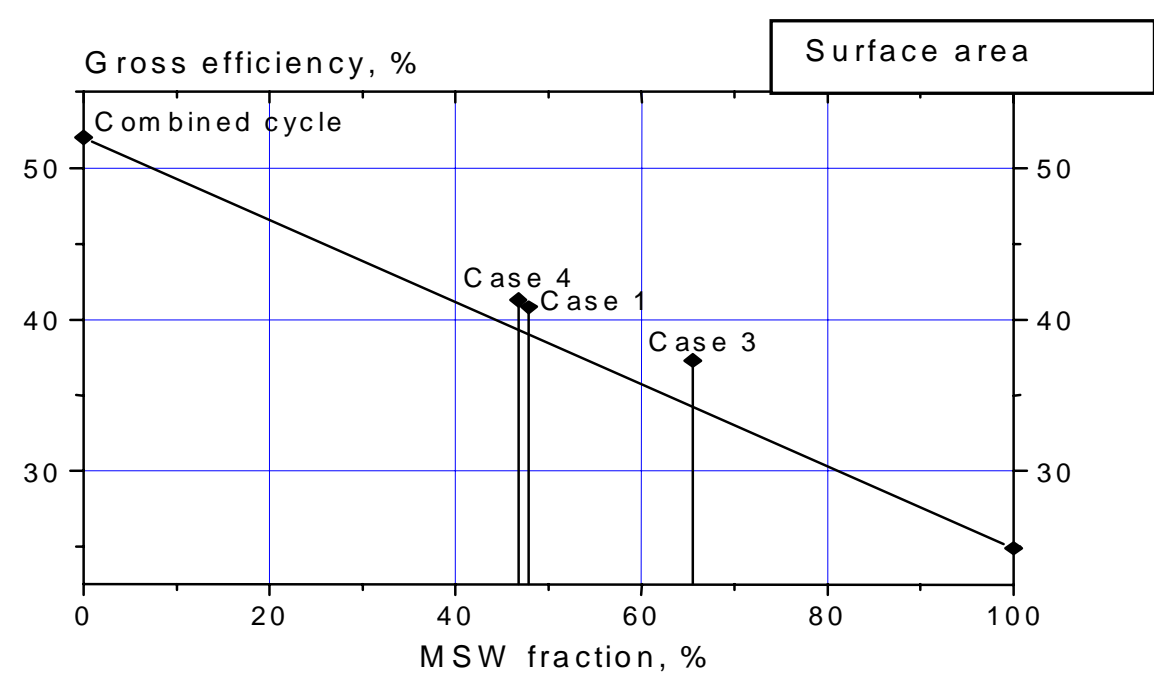

Figure 7 Exergy diagram of the life cycle of the porcelain mug

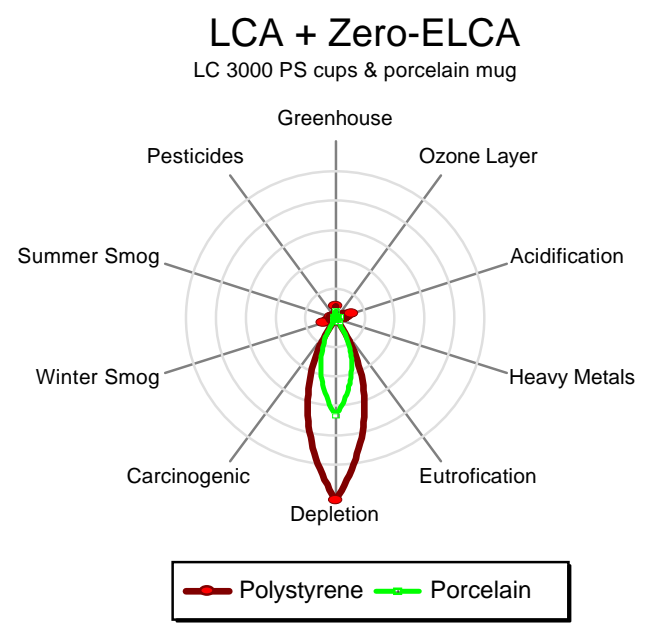

Figure 8 The normalised environmental effects in the Zero-ELCA

The conclusion is that an exergetic life cycle analysis (ELCA) gives a correct estimate of the depletion of natural resources. It is not a good quantifier for other environmental effects as these effects can be reduced drastically by suitably spending more exergy.

The factor of 2 for the exergy loss of the cup and the mug should be judged with modesty. The greater exergy loss of the cup depends on many assumptions. People that use their cup twice and do not wash up are all right. They spend as much exergy as mug users. 


\section{RECYCLING}

Without changing people's personal, hygienic habits, a reduction of the exergy loss associated with using cups can be achieved tentatively by recycling the polystyrene. Naturally recycling only makes sense when the recycling effort and the loss of electricity from incineration are considerably smaller than making fresh polystyrene. This can be reformulated and yield a more general rule: recycling of a product requires an effort in terms of exergy that should be small with respect to the making of the product minus its incineration. By doing so it can be judged which option is best as far as the depletion of fossil fuels is concerned.

During this conference the interaction of recycling and the use of renewable materials as fuel or feed stock is dealt with. The conclusion is that we need methodology for judging such an interaction. Without methodology we can not formulate clear conclusions with respect to sustainability.

\section{THERMO-ECONOMICS}

In the future thermo-economic methods should be extended to include an exergy analysis of the first and the third phase of the life cycle of a product or a plant. Thus, it should include exergy investment and exergy disposal. The frequently mentioned excuse that the exergy loss is small during these phases is not generally true. Moreover, the objective of thermo-economic analysis is to find a compromise between monetary and exergetic cost. In view of future sustainability for industry and its products it is desirable to weigh a potentially modest increase of exergy investment against a potentially large reduction of exergy loss during operation by comparing the consequences in terms of monetary cost.

An added advantage of exergy analysis of plants, components and products in the first and third phase of the life cycle is the fact that cost engineers do not have support from the scientific community. A cost engineer is one of the few types of engineers in the world in need of a scientifically sound theory. By developing exergy analysis for cost engineers and by somehow correlating the exergy results with monetary cost might be helpful. An important aspect is the incorporation of human labor into exergetic and monetary cost.

\section{NEW FIELDS FOR THERMODYNAMICS}

Thermodynamics is conspicuously absent from a number of fields in our society. The reason is on the one hand that thermodynamics is not easily understood and on the other hand that teaching thermodynamics appears to be difficult. It is not easy to explain the second law to:

- economists, both macro and micro

- civil servants with a degree in public administration,

- environmental engineers,

- lawyers,

- farmers,

- architects,

- engineers of town and country planning and

- accountants.

Nevertheless, all of them are busy imitating thermodynamic concepts:

- economists are trying to internalize external cost 
- civil servants with a degree in public administration are studying the effectiveness of subsidized energy conservation

- environmental engineers are desparately looking for the CO2-effect

- lawyers are trying to differentiate between crude oil used as a basis for fuel and feedstock

- farmers would like vegetarians to eat meat and so increase the use of natural fertilizer

- architects want to know whether concrete beams are sustainable

- engineers of town and country planning minimize the area per one-family-house

- accountants check environmental reports

Many of them would be delighted to become experts in thermodynamics if convinced that thermodynamics could be useful to them. As usual this will not happen by us convincing them. It will happen gradually and automatically. The reason can be found in the fact that we have found a common, new and optimistic view of the future. We would like the future to be sustainable. Sustainability is a concept in need of accountability. Sustainability will be made accountable by thermodynamics.

\section{CONCLUSIONS}

Sustainability is a concept that diverts from the focus on one or several specific subjects in environmental affairs to the environment as a whole. In the past the focus has switched swiftly from radiation to nuclear waste, from the ozone layer to cigarette smoke and from acidification to the green house effect. The problem with this kind of focus is that a long term environmental policy is absent. If the greenhouse effect is not as serious as now predicted, international treaties are obsolete. Therefore, sustainability is a better concept. Thermodynamics is sufficiently basic and general to make it accountable.

\section{REFERENCES}

Cornelissen, R.L., 1997, Thermodynamics and sustainable development. PhD-thesis, University of Twente, The Netherlands.

Heijungs, R., 1992, Environmental life cycle assessment of products, Guide. Centre of Environmental Science, Leiden, The Netherlands.

Heijungs, R., 1992, Environmental life cycle assessment of products, Backgrounds. Centre of Environmental Science, Leiden, The Netherlands.

Korobitsyn, M.A., 1998, Analysis of Cogeneration, Combined and Integrated Cycles. PhDthesis, University of Twente, The Netherlands.

Udo de Haes, H.A., 1996, Towards a methodology for life cycle impact assessment Society of Environmental Toxicology and Chemistry - Europe Brussels, Belgium, September. 